\title{
IR photometric properties of Red Giants in $\omega$ Cen $\star$
}

\author{
A. Sollima ${ }^{1,2}$, F. R. Ferraro ${ }^{1}$, L. Origlia ${ }^{2}$, E. Pancino ${ }^{2}$, and M. Bellazzini ${ }^{2}$ \\ 1 Dipartimento di Astronomia, Università di Bologna, via Ranzani 1, 40127 Bologna, Italy \\ e-mail: ferraro@bo.astro.it; s_sollima@astbo4.bo.astro.it \\ 2 Osservatorio Astronomico di Bologna, via Ranzani 1, 40127 Bologna, Italy
}

Received 5 November 2003 / Accepted 27 January 2004

\begin{abstract}
We present a near-infrared $J$ and $K$ photometric catalog containing more than 73000 stars in the central region of the giant Globular Cluster $\omega$ Centauri ${ }^{\star \star}$. This is the largest IR data set ever published for this cluster and has been used to completely characterize the morphology and the properties of the Red Giant Branch (RGB). In particular, we concentrated our attention on (i) the anomalous RGB (RGB-a), recently discovered in this cluster and (ii) the RGB of the dominant metal poor population (RGB-MP) in both the infrared $(K, J-K)$ and optical-infrared $(K, V-K)$ color magnitude diagrams. The full set of morphological parameters and photometric indices has been measured and compared with the empirical relations by Ferraro et al. (2000). We find that the detailed photometric properties of the RGB-a are in full agreement with the recent spectroscopic metallicity estimates that place it at the metal-rich extreme of the stellar population mix in $\omega$ Centauri.
\end{abstract}

Key words. globular clusters: individual: $\omega$ Cen - stars: Population II - stars: evolution - techniques: photometric - infrared: stars

\section{Introduction}

The origin and star formation history in $\omega$ Centauri, the most luminous and massive globular cluster in our Galaxy, is one of the most intriguing problems of modern stellar astrophysics. $\omega$ Centauri is the only known Galactic globular cluster which shows clear variations in the metal content of its giants. This evidence has been firmly estabilished in the past by extensive low (Norris et al. 1996; Suntzeff \& Kraft 1996) and high resolution (Norris \& Da Costa 1995; Smith et al. 2000) spectroscopic surveys. More recently, the scenario has become more complicated due to the discovery of an additional, metal-rich population with its own distinct RGB (hereafter RGB-a) that contains approximately $5 \%$ of the red giants in $\omega$ Cen (Lee et al. 1999; Pancino et al. 2000, 2002). In spite of the huge observational effort carried out so far, the global picture of the cluster formation and evolution is far from being completely understood.

In this framework, we have started a long-term project devoted to the detailed study of the properties of the different stellar populations in this cluster (see the overview of the project by Ferraro et al. 2001). Within this project a number of

Send offprint requests to: A. Sollima,

e-mail: s_sollima@astbo4.bo.astro.it

* Based on UVES observations collected at the European Southern Observatory, La Silla, Chile, within the observing programmes 64.N-0038 and 68.D-0287.

$\star \star$ The entire IR catalog is only available in electronic form at the CDS via anonymous ftp to

cdsarc.u-strasbg.fr $(130.79 .128 .5)$ or via

http://cdsweb.u-strasbg.fr/cgi-bin/qcat?]/A+A/420/173 results have been published, in particular on the identification of the anomalous RGB-a, and on the definition of its chemical and kinematic properties (see Pancino et al. 2000, 2002, 2003; Bellazzini et al. 2001; Ferraro et al. 2002; Origlia et al. 2002). Most of the actual observational knowledge comes from the optical (photometric or spectroscopic) study of RGB stars. Only sparse literature of near infrared observations existed up to now. Two pioneering studies by Glass \& Feast $(1973,1977)$ and Persson et al. (1980) present $J, H$ and $K$ magnitudes for a few tens of bright giants. More recently, the NICMOS camera on board of HST has been used by Pulone et al. (1998) to obtain extremely deep photometry of a tiny area $\left(20^{\prime \prime} \times 20^{\prime \prime}\right) 7$ arcmin away from the cluster center. The 2MASS survey has instead covered a very wide area $\left(3^{\circ} \times 2^{\circ}\right)$ around $\omega$ Cen, which however does not include the central region of the cluster. This paper presents a large $J$ and $K$ photometric catalog of more than 73000 stars in an area covering $\sim 13^{\prime} \times 13^{\prime}$ around the center of $\omega$ Cen. By combining the IR-data set with wide field optical photometry (Pancino et al. 2000, 2003), we measured the complete set of morphological parameters defined by Ferraro et al. (2000, hereafter F00), which fully characterize the photometric properties of the RGB of different populations in $\omega$ Centauri.

\section{Observational material}

A large set of $J$ and $K$ images were obtained at ESO, La Silla (Chile), during two observing runs (Run \#1 and \#2, see Table 1) at the New Technology Telescope (NTT) using the near-IR imager/spectrometer SOFI. The observations were secured as back-up programmes. SOFI is an imager/spectrometer 


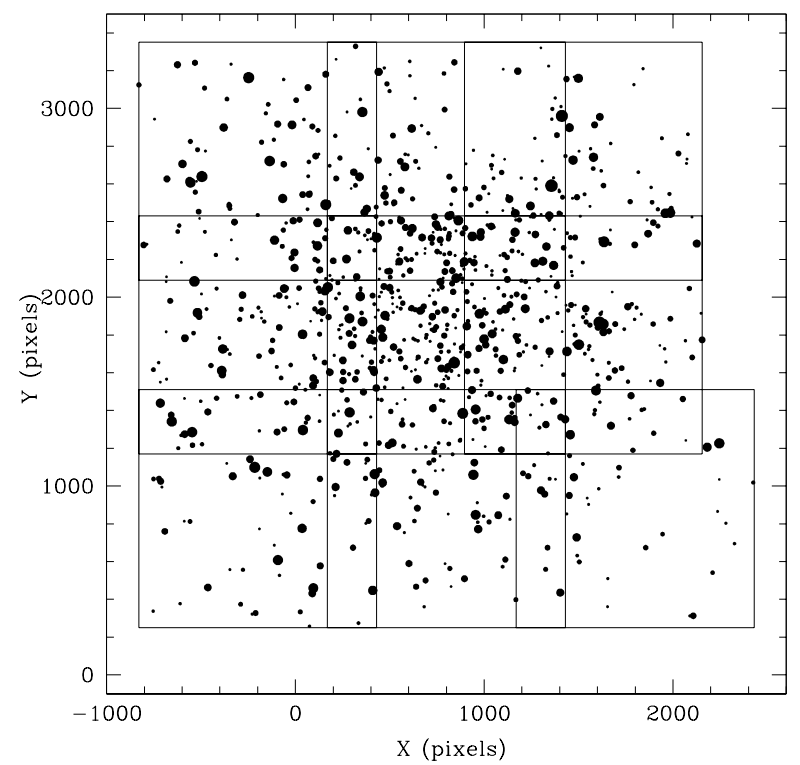

Fig. 1. Map of the region sampled by the IR observations. North is up, east on the left. Only stars with $K<13$ have been plotted.

Table 1. Observing logs.

\begin{tabular}{cccc}
\hline \hline Run & Date & Filter & NDIT $\times$ DIT \\
\hline 1 & 13-14 Jan. 2000 & $J$ & $45 \times 1.2$ \\
1 & 13-14 Jan. 2000 & $K_{\mathrm{s}}$ & $150 \times 1.2$ \\
1 & 13-14 Jan. 2000 & $J$ & $15 \times 1.2$ \\
1 & 13-14 Jan. 2000 & $K_{\mathrm{s}}$ & $60 \times 1.2$ \\
2 & 30-31 Dec. 2001 & $J$ & $60 \times 1.2$ \\
2 & 30-31 Dec. 2001 & $K_{\mathrm{s}}$ & $120 \times 1.2$ \\
\hline
\end{tabular}

equipped with a $1024 \times 1024$ Rockwell IR-array detector. All the observations presented here were performed with a scale of $0.292^{\prime \prime} /$ pixel, fixing a global FoV of $\sim 5^{\prime} \times 5^{\prime}$ each field.

During Run\#1, we covered the central region of the cluster with a mosaic of nine partially overlapping fields (hereafter Science fields), sampling a total area of $\sim 13^{\prime} \times 13^{\prime}$ around the cluster center (see Fig. 1). A set of high-S/N flatfields in each filter has been obtained with a halogen lamp, alternately switched on and off. For each field observed in the cluster, a corresponding sky field has also been observed ( 10 arcmin away from the cluster center) using the same instrument configuration. Each sky image was obtained as the median of at least 5 frames, shifted by some hundreds of pixels with respect to each other. The final set consists of 18 sky-subtracted and flatfield corrected images, 9 for each filter.

During Run\#2 a set of 4 additional fields (hereafter Calibration fields), entirely within the area sampled in Run\#1, has been obtained for photometric calibration purposes. The same pre-reduction procedure used for the Science fields has been also applied to the Calibration fields.

All photometric reductions were carried out using ROMAFOT (Buonanno et al. 1983). The details of the reduction procedure have already been reported in previous papers (Ferraro et al. 1994, 1995). We just mention here that for the IR frames we used the PSF-fitting routine specifically modified to deal with under-sampled stellar images
(Buonanno \& Iannicola 1988). The source detection was performed independently on each field; more than 80000 separate stars have been detected in total in the nine science fields observed during Run\#1.

\subsection{Photometric calibration}

During Run\#2, a set of nine standard stars from the Persson et al. (1998) list was observed together with the 4 Calibration fields in the cluster. Five measurements for each standard were secured and averaged, for each filter. The calibrating equations linking the aperture photometry to the standard system are:

$K=k+22.30 \pm 0.02$

$J=j+23.03 \pm 0.02$

where $j$ and $k$ are the instrumental magnitudes, $J$ and $K$ the calibrated ones. The existence of a slight residual colour equation (with slope $<0.01$ ) in both filters cannot be totally excluded.

The two above equations have been used to calibrate the 4 Calibration fields in $\omega$ Cen: as usual the most isolated and brightest stars in the field have been employed to link the aperture magnitudes to the fitting instrumental magnitudes, after normalizing for exposure time and correcting for airmass. A catalog with more than 10000 calibrated stars in the cluster has been produced that has been used to calibrate the global IR catalog, including more than 73000 stars.

Our calibration was compared with the previous photometric catalogues. Unfortunately, only 8 stars were found in common between the present catalog and the pioneering work by Persson et al. (1980), while no star is in common with the very deep study by Pulone et al. (1998). The average magnitude differences found are $\Delta J=0.034 \pm 0.063$ and $\Delta K=$ $0.093 \pm 0.079$, fully consistent with there being no systematic offset between our catalog and the one by Persson et al. (1980) within the errors. A much more significant comparison can be achieved with the 2MASS catalog ${ }^{1}$ (see also Sect. 5), showing that our calibrated magnitudes are in very good agreement with the 2MASS photometric system $(\Delta J=0.00 \pm 0.10$ and $\Delta K=-0.04 \pm 0.10)$.

\subsection{Astrometry and resulting catalog}

The photometrically calibrated catalog has been compared with the large proper motion catalog by van Leeuwen et al. (2000), which lists accurate positions for more than 10000 red giants in $\omega$ Cen. We used a software package under development at the Bologna Observatory (Montegriffo et al. 2004, in preparation), specifically designed to correlate populous and crowded stellar catalogs, and to obtain precise astrometry of large fields. We thus were able to produce coordinates in the J2000 reference frame for stars in the whole catalog, with a typical rms of 200 mas.

The final on-line catalog format and content is illustrated in Table 2. For each star we present, along with the star number and coordinates, the $J$ and $K$ magnitudes and their errors, obtained as the standard deviation from the mean of repeated

${ }^{1}$ See Cutri et al. (2003), Explanatory Supplement to the 2MASS All Sky Data Relase, http://www.ipac.caltech.edu/2mass/ 

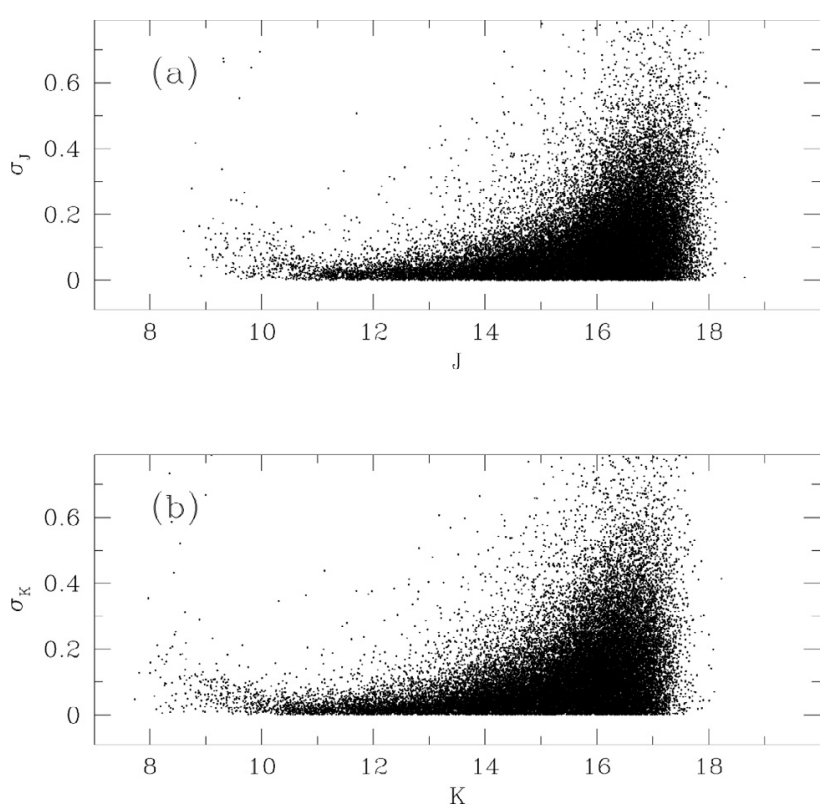

Fig. 2. $J$ (panel a)) and $K$ (panel b)) photometric errors as a function of magnitude, represented by the standard deviation from the mean of repeated measurements.

Table 2. A sample of the online catalog. Only a few entries are displayed to illustrate the catalog format and contents. See text for a detailed description on the measurement procedures.

\begin{tabular}{ccccccc}
\hline \hline Id & $\begin{array}{c}J \\
(\mathrm{mag})\end{array}$ & $\begin{array}{c}K \\
(\mathrm{mag})\end{array}$ & $\begin{array}{c}\sigma_{J} \\
(\mathrm{mag})\end{array}$ & $\begin{array}{c}\sigma_{K} \\
(\mathrm{mag})\end{array}$ & $\begin{array}{c}\mathrm{RA} \\
(\mathrm{deg})\end{array}$ & $\begin{array}{c}\text { Dec } \\
(\mathrm{deg})\end{array}$ \\
\hline 1 & 8.728 & 7.731 & 0.043 & 0.047 & 201.7852918 & -47.5302560 \\
2 & 9.178 & 8.291 & 0.100 & 0.038 & 201.7787851 & -47.5318524 \\
3 & 9.134 & 8.103 & 0.063 & 0.135 & 201.7231633 & -47.5327578 \\
4 & 9.274 & 8.305 & 0.077 & 0.183 & 201.7602655 & -47.4837799 \\
5 & 9.305 & 8.401 & 0.070 & 0.059 & 201.7608180 & -47.5516698 \\
\hline
\end{tabular}

measurements: when a star was measured only once, we assigned a null error value to its magnitude. Figure 2 shows the typical photometric errors for the $J$ and $K$ bands, as a function of the magnitude. The mean error at the Horizontal Branch $(\mathrm{HB})$ magnitude level $(J \simeq 14.2, K \simeq 14)$ is about $0.02 \mathrm{mag}$ in both bands. Figure 3 presents the $(K, J-K)$ color Magnitude Diagram (CMD) obtained from the final IR catalog.

The IR catalog has also been correlated with the partially published wide field optical catalog by Pancino et al. (2000, 2003). A final multi-wavelength catalog ( $B, V, I, J$ and $K$ ) has been obtained and will be used throughout the paper. As an example of combined optical-infrared CMD, Fig. 4 shows the $(K, B-K)$ CMD.

\subsection{Colour magnitude diagrams}

The main features of the CMDs presented in Figs. 3 and 4 are listed below:

1. the CMDs sample the entire evolved populations in the cluster, reaching the TO region $(K \sim 17)$;

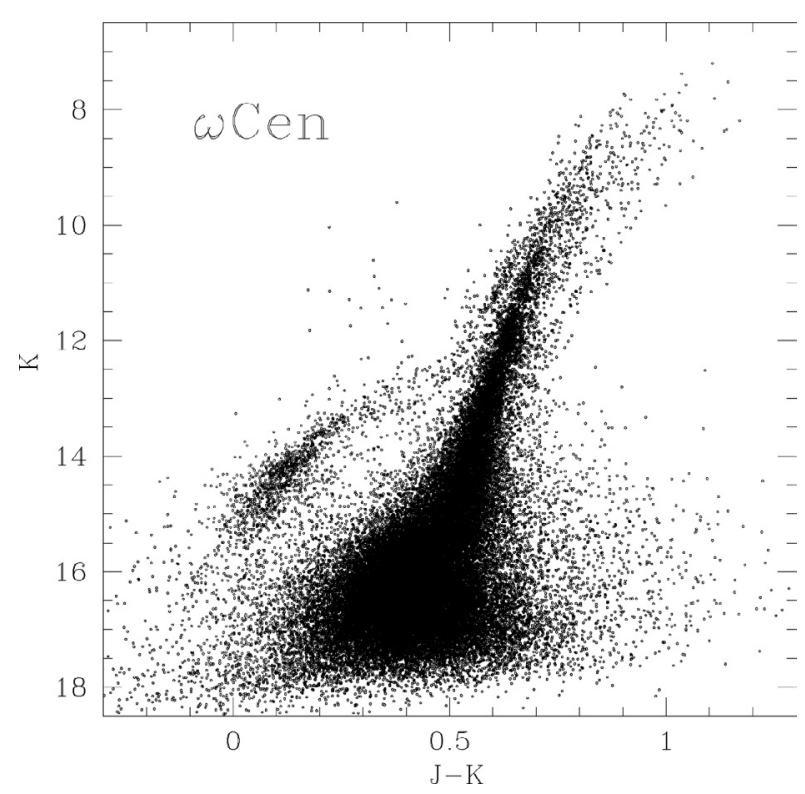

Fig. 3. $(K, J-K)$ color magnitude diagram for $\sim 73000$ stars measured in $\omega$ Cen.

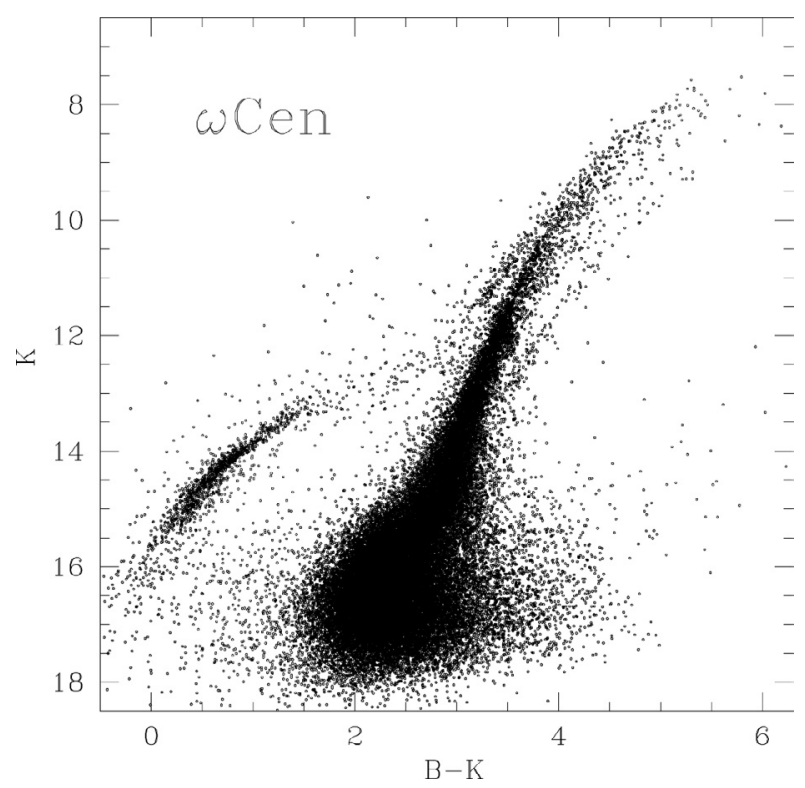

Fig. 4. The combined $(K, B-K) \mathrm{CMD}$ for stars detected in $\omega$ Cen. The $B$ magnitudes are from Pancino et al. (2000).

2. the complex structure of the RGB in $\omega$ Cen is clearly visible, starting from the blue side, where the dominant metalpoor population defines the main RGB (RGB-MP);

3. the anomalous RGB (RGB-a, as defined by Pancino et al. 2000), is clearly visible as a well separated population at the extreme red side of the main RGB structure, having $K<13$ and $(J-K)>0.6$ (see Fig. 3$)$ and $(B-K)>3.4$ (see Fig. 4);

4. an intermediate RGB component (RGB-MInt), which uniformly populates the CMD region between the RGB-MP and the RGB-a, can also be easily noticed;

5. the AGB stars appear well separated from the RGB in both CMDs;

6. the HB is well defined $((B-K)<2)$; in particular, a significant population of under-luminous HB stars 


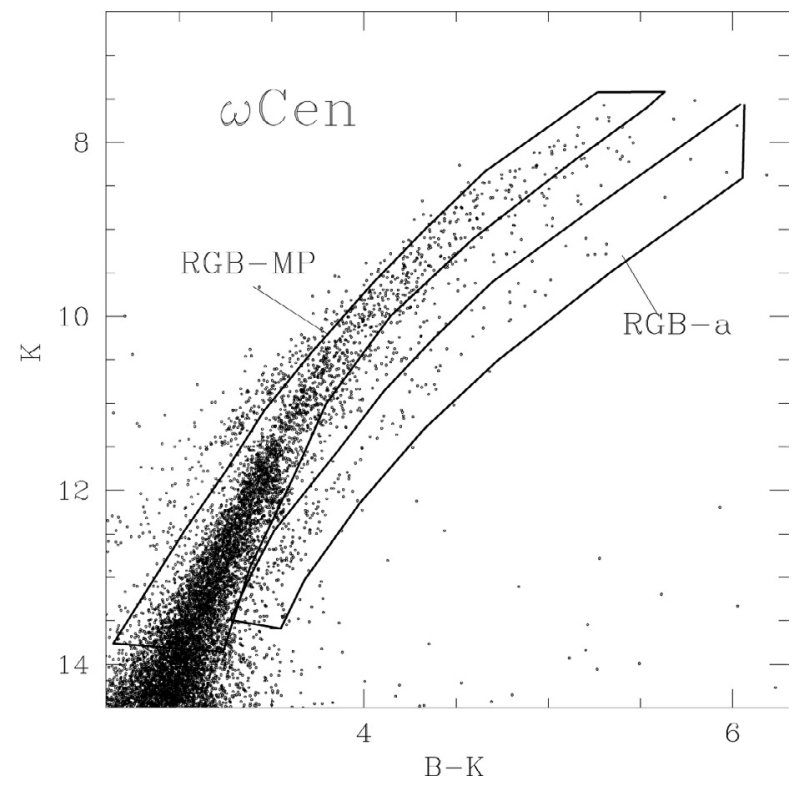

Fig. 5. The zoomed CMD of the RGB region. Selection boxes for the metal poor (RGB-MP) and anomalous (RGB-a) population are shown.

(D'Cruz et al. 1999) is visible at blue colours $((B-K)<1)$, lying below the main HB locus.

The dominant RGB-MP component is extremely well populated, allowing a robust measurement of the RGB-tip (Bellazzini et al. 2004, in preparation) and the RGB bump position. In the following, we will discuss the photometric properties and the evolutionary features of the two best defined RGB populations: the RGB-MP and the RGB-a, since the intermediate population is spread within these two extremes.

\section{RGB fiducial lines}

The first step to study the photometric properties of the two populations is the determination of the mean ridge lines. To do this, we applied the standard procedure, already described in Ferraro at al. (1999, 2000).

As usual, a first selection was made by eye. In particular, in the case of RGB-MP, we took special care in excluding $\mathrm{HB}$ and $\mathrm{AGB}$ stars, which are easily identified from the high quality CMDs shown in Figs. 3 and 4. The selection of the RGB samples could in principle be disturbed by the presence of the RGB-MInt stars. However, RGB-a stars are well separated from the remaining populations when selected from the optical-infrared CMD of Fig. 4. The contamination of the RGB-MP population from the RGB-MInt stars can be quantified to be $<10 \%$, with negligible impact on the ridge line determination of the former. Figure 5 shows the adopted selection boxes for the RGBs of the different populations. We used a low-order polynomial to fit the selected stars and an iterative procedure to automatically reject stars lying more than $2 \sigma$ away from the best-fit line. The iteration was continued until convergence to a stable fit was obtained. The resulting mean ridge lines in various planes are reported in Table 3 (for the RGB-MP) and Table 4 (for the RGB-a).
Table 3. Mean ridge line of the RGB-MP in $\omega$ Cen.

\begin{tabular}{ccccc}
\hline \hline$K$ & $J-K$ & $V-K$ & $B-K$ & $B-J$ \\
\hline 7.8 & 0.90 & 3.74 & 5.24 & 4.26 \\
8.0 & 0.88 & 3.63 & 5.09 & 4.14 \\
8.2 & 0.86 & 3.54 & 4.96 & 4.03 \\
8.4 & 0.85 & 3.45 & 4.82 & 3.93 \\
8.6 & 0.83 & 3.37 & 4.70 & 3.83 \\
8.8 & 0.81 & 3.29 & 4.58 & 3.73 \\
9.0 & 0.80 & 3.21 & 4.47 & 3.64 \\
9.2 & 0.78 & 3.14 & 4.36 & 3.56 \\
9.4 & 0.77 & 3.08 & 4.27 & 3.48 \\
9.6 & 0.76 & 3.02 & 4.17 & 3.40 \\
9.8 & 0.74 & 2.96 & 4.08 & 3.34 \\
10.0 & 0.72 & 2.90 & 4.00 & 3.26 \\
10.2 & 0.72 & 2.85 & 3.92 & 3.20 \\
10.4 & 0.71 & 2.82 & 3.85 & 3.14 \\
10.6 & 0.70 & 2.76 & 3.78 & 3.08 \\
10.8 & 0.69 & 2.72 & 3.71 & 3.02 \\
11.0 & 0.68 & 2.68 & 3.65 & 2.97 \\
11.2 & 0.67 & 2.64 & 3.60 & 2.92 \\
11.4 & 0.66 & 2.60 & 3.54 & 2.88 \\
11.6 & 0.65 & 2.57 & 3.49 & 2.83 \\
11.8 & 0.64 & 2.54 & 3.44 & 2.79 \\
12.0 & 0.63 & 2.51 & 3.39 & 2.75 \\
12.2 & 0.62 & 2.48 & 3.35 & 2.71 \\
12.4 & 0.61 & 2.45 & 3.31 & 2.68 \\
12.6 & 0.61 & 2.42 & 3.27 & 2.64 \\
12.8 & 0.60 & 2.39 & 3.23 & 2.61 \\
13.0 & 0.59 & 2.36 & 3.19 & 2.58 \\
13.2 & 0.58 & 2.33 & 3.15 & 2.54 \\
13.4 & 0.58 & 2.30 & 3.11 & 2.51 \\
13.6 & 0.57 & 2.27 & 3.08 & 2.48 \\
13.8 & 0.56 & 2.24 & 3.04 & 2.45 \\
14.0 & 0.56 & 2.20 & 3.00 & 2.42 \\
14.2 & 0.55 & 2.17 & 2.96 & 2.39 \\
14.4 & 0.54 & 2.13 & 2.93 & 2.36 \\
14.6 & 0.54 & 2.09 & 2.89 & 2.33 \\
\hline & & & &
\end{tabular}

To convert the derived RGB mean ridge lines to the absolute $M_{K},(J-K)_{0}$ and $M_{K},(V-K)_{0}$ planes and to measure the photometric indices and morphology parameters defined by F00 (see Sect. 4), we need to make some basic assumptions for the metallicity, reddening and distance for the subpopulations of $\omega$ Cen.

\subsection{Metallicity}

For the dominant metal-poor (RGB-MP) population we adopted the metallicity obtained by the most extensive spectroscopic survey performed in $\omega$ Cen by Norris et al. (1996), who provided a peak value of $[\mathrm{Ca} / \mathrm{H}]=-1.4 \pm 0.1$. Using the stars in our sample which have both $[\mathrm{Ca} / \mathrm{H}]$ from Norris et al. (1996) and $[\mathrm{Fe} / \mathrm{H}]$ abundances from Suntzeff \& Kraft (1996), we translated the $[\mathrm{Ca} / \mathrm{H}]$ peak assumed above into $[\mathrm{Fe} / \mathrm{H}] \sim-1.6 \pm 0.1$. Both high-resolution optical spectra (Pancino et al. 2002) and medium-resolution IR spectra (Origlia et al. 2003) suggest a significantly higher metallicity for the anomalous (RGB-a) population, $[\mathrm{Fe} / \mathrm{H}]=-0.6 \pm 0.15$. 
Table 4. Mean ridge line of the RGB-a in $\omega$ Cen.

\begin{tabular}{ccccc}
\hline \hline$K$ & $J-K$ & $V-K$ & $B-K$ & $B-J$ \\
\hline 8.2 & 1.15 & 4.49 & 6.00 & 5.10 \\
8.4 & 1.12 & 4.34 & 5.84 & 4.92 \\
8.6 & 1.09 & 4.20 & 5.53 & 4.59 \\
8.8 & 1.06 & 4.06 & 5.53 & 4.59 \\
9.0 & 1.03 & 3.94 & 5.39 & 4.42 \\
9.2 & 1.01 & 3.82 & 5.25 & 4.30 \\
9.4 & 0.98 & 3.72 & 5.12 & 4.18 \\
9.6 & 0.96 & 3.61 & 5.00 & 4.06 \\
9.8 & 0.93 & 3.52 & 4.87 & 3.94 \\
10.0 & 0.91 & 3.43 & 4.76 & 3.84 \\
10.2 & 0.89 & 3.35 & 4.65 & 3.74 \\
10.4 & 0.87 & 3.27 & 4.54 & 3.65 \\
10.6 & 0.85 & 3.20 & 4.44 & 3.56 \\
10.8 & 0.83 & 3.13 & 4.34 & 3.48 \\
11.0 & 0.81 & 3.07 & 4.24 & 3.41 \\
11.2 & 0.79 & 3.01 & 4.15 & 3.33 \\
11.4 & 0.78 & 2.95 & 4.06 & 3.27 \\
11.6 & 0.76 & 2.90 & 3.98 & 3.20 \\
11.8 & 0.74 & 2.84 & 3.90 & 3.14 \\
12.0 & 0.73 & 2.79 & 3.82 & 3.08 \\
12.2 & 0.71 & 2.74 & 3.74 & 3.02 \\
12.4 & 0.70 & 2.69 & 3.67 & 2.96 \\
12.6 & 0.68 & 2.64 & 3.60 & 2.90 \\
12.8 & 0.67 & 2.59 & 3.53 & 2.84 \\
13.0 & 0.65 & 2.54 & 3.46 & 2.78 \\
13.2 & 0.64 & 2.49 & 3.40 & 2.72 \\
13.4 & 0.63 & 2.43 & 3.34 & 2.65 \\
13.6 & 0.61 & 2.37 & 3.27 & 2.59 \\
\hline & & & &
\end{tabular}

Straniero \& Chieffi (1991) and Salaris et al. (1993) showed that, when computing the isochrones of Population II stars, the contribution of the $\alpha$-element enhancement can be taken into account by simply rescaling standard models to the global metallicity $[\mathrm{M} / \mathrm{H}]$, according to the following relation

$[\mathrm{M} / \mathrm{H}]=[\mathrm{Fe} / \mathrm{H}]+\log _{10}\left(0.638 f_{\alpha}+0.362\right)$

where $f_{\alpha}$ is the enhancement factor for the $\alpha$-elements. We assumed $[\alpha / \mathrm{Fe}] \simeq+0.3$ for the RGB-MP (Norris \& Da Costa 1995; Smith et al. 2000) and a lower enhancement $[\alpha / \mathrm{Fe}] \simeq+0.1$ for the RGB-a, following Pancino et al. (2002) and Origlia et al. (2003). Thus, we obtained a global metallicity of $[\mathrm{M} / \mathrm{H}]=-1.39 \pm 0.10$ and $[\mathrm{M} / \mathrm{H}]=-0.53 \pm 0.21$ for the RGB-MP and the RGB-a, respectively.

\subsection{ZAHB level}

To convert the fiducial ridge lines into the absolute plane, we need to assume a distance modulus and a reddening correction. In the following, we adopt the distance scale presented by Ferraro et al. (1999), which is based on the comparison between the actual level of the Zero Age Horizontal Branch (ZAHB) and the theoretical models computed by Straniero et al. (1997). Using the optical photometric catalog partially published by Pancino et al. (2000) and following the prescriptions of Ferraro et al. (1999),

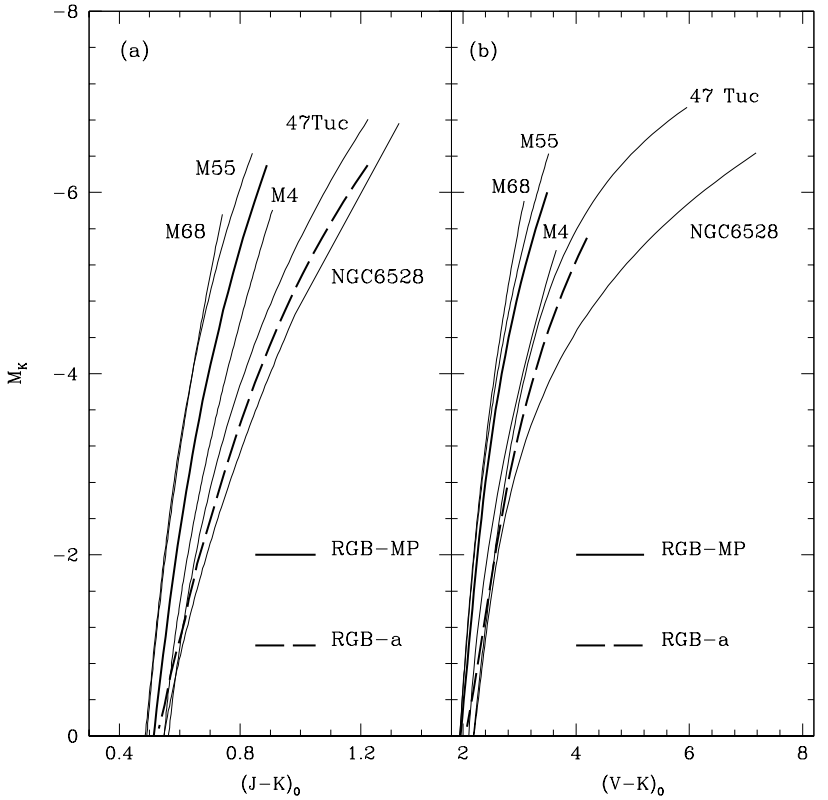

Fig. 6. Fiducial ridge lines in the $M_{K},(J-K)_{0}$ plane (panel a)) and in the $M_{K},(V-K)_{0}$ plane (panel b)) for a few GGCs of F00 and for the two populations of $\omega$ Cen.

we obtain for $\omega$ Cen $V_{\text {ZAHB }}=14.55 \pm 0.09$. Assuming a metallicity of $[\mathrm{Fe} / \mathrm{H}]=-1.6$ for the main population (see above) and following Eq. (4) of Ferraro et al. (1999), we obtain $M_{V}^{\mathrm{ZAHB}}=0.56 \pm 0.09$. Adopting $E(B-V)=0.11 \pm 0.01$ (Lub $2002)$, the distance modulus finally turns out to be $(m-M)_{0}=$ $13.65 \pm 0.13$, in nice agreement with the most recent determinations by Thompson et al. (2001) and Caputo et al. (2002).

\subsection{Comparison with the FOO sample}

The ridge lines of Tables 3 and 4 have been transformed to the absolute planes $M_{K},(J-K)_{0}$ and $M_{K},(V-K)_{0}$, using the distance modulus and the reddening discussed above, and assuming $A_{J}=0.87 E(B-V)$ and $A_{K}=0.38 E(B-V)$ extinction coefficients in the $J$ and $K$ bands, respectively (Savage \& Mathis 1979). Figure 6 compares the ridge lines of $\omega$ Cen with the lines of a few globular clusters belonging to the sample presented by F00.

In both planes the RGB-MP ridge line is located between the metal-poor clusters $\mathrm{M} 68([\mathrm{Fe} / \mathrm{H}] \sim-1.99)$ and $\mathrm{M} 4$ $([\mathrm{Fe} / \mathrm{H}] \sim-1.19)$, close to M $55([\mathrm{Fe} / \mathrm{H}] \sim-1.61)$ as expected, given its metallicity. The RGB-a is located between the metal-rich clusters 47 Tuc $([\mathrm{Fe} / \mathrm{H}] \sim-0.7)$ and NGC 6528 $([\mathrm{Fe} / \mathrm{H}] \sim-0.38)$, in good agreement with the spectroscopic constraints.

\section{RGB colors and morphology}

In order to quantitatively describe the morphology and properties of the RGBs of the different populations in $\omega$ Cen, we measured the full set of photometric parameters defined by F00. 


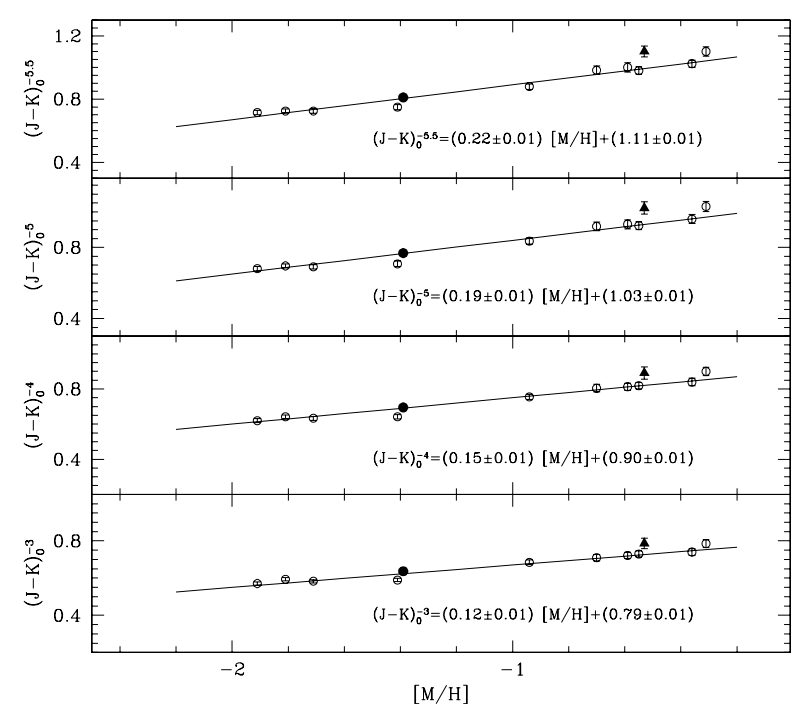

Fig. 7. RGB mean $(J-K)_{0}$ colors at different magnitudes $\left(M_{K}=\right.$ $-3,-4,-5,-5.5)$ as a function of global metallicity for the ten GGCs of F00 (open circles), the RGB-MP (filled circle) and the RGB-a (filled triangle).

Table 5. Inferred $(J-K)_{0}$ RGB colors at fixed magnitudes.

\begin{tabular}{ccccc}
\hline \hline & $(J-K)_{0}^{-5.5}$ & $(J-K)_{0}^{-5}$ & $(J-K)_{0}^{-4}$ & $(J-K)_{0}^{-3}$ \\
\hline RGB-MP & $0.81 \pm 0.02$ & $0.77 \pm 0.02$ & $0.69 \pm 0.02$ & $0.64 \pm 0.02$ \\
RGB-a & $1.10 \pm 0.03$ & $1.02 \pm 0.03$ & $0.89 \pm 0.04$ & $0.79 \pm 0.03$ \\
\hline
\end{tabular}

Table 6. Inferred $(V-K)_{0}$ RGB colors at fixed magnitudes.

\begin{tabular}{ccccc}
\hline \hline & $(V-K)_{0}^{-5.5}$ & $(V-K)_{0}^{-5}$ & $(V-K)_{0}^{-4}$ & $(V-K)_{0}^{-3}$ \\
\hline RGB-MP & $3.24 \pm 0.03$ & $3.03 \pm 0.03$ & $2.69 \pm 0.03$ & $2.44 \pm 0.03$ \\
RGB-a & $4.19 \pm 0.21$ & $3.83 \pm 0.20$ & $3.27 \pm 0.09$ & $2.87 \pm 0.08$ \\
\hline
\end{tabular}

The main RGB observables measured in the IR CMDs are the following:

- the RGB $(J-K)_{0}$ and $(V-K)_{0}$ colors at different magnitude levels;

- the RGB $M_{K}$ magnitude at fixed colors;

- the RGB slope.

In Tables 5 and 6 , the inferred $(J-K)_{0}$ and $(V-K)_{0}$ colors at fixed $M_{K}$ magnitudes are listed. Our results are in good agreement with the empirical relations proposed by F00 in both colors and magnitudes, the $(J-K)_{0}$ colors of the RGB-a ridge line being only slightly redder than predicted, but still within the overall uncertainties.

Following F00, we also measured the absolute $M_{K}$ magnitude at $(V-K)_{0}=3$ and $(J-K)_{0}=0.7$. In Fig. 9 the dependence of these two parameters on global metallicity is shown; the measurements taken on the RGB-MP and RGB-a mean lines of $\omega$ Cen are overplotted as solid symbols. At $(V-K)_{0}=3$, we observe a nice agreement between the magnitudes measured for both the RGB-MP and RGB-a populations and the relations proposed by F00. At $(J-K)_{0}=0.7$, the absolute $M_{K}$ magnitudes observed for the two RGB populations of $\omega$ Cen are

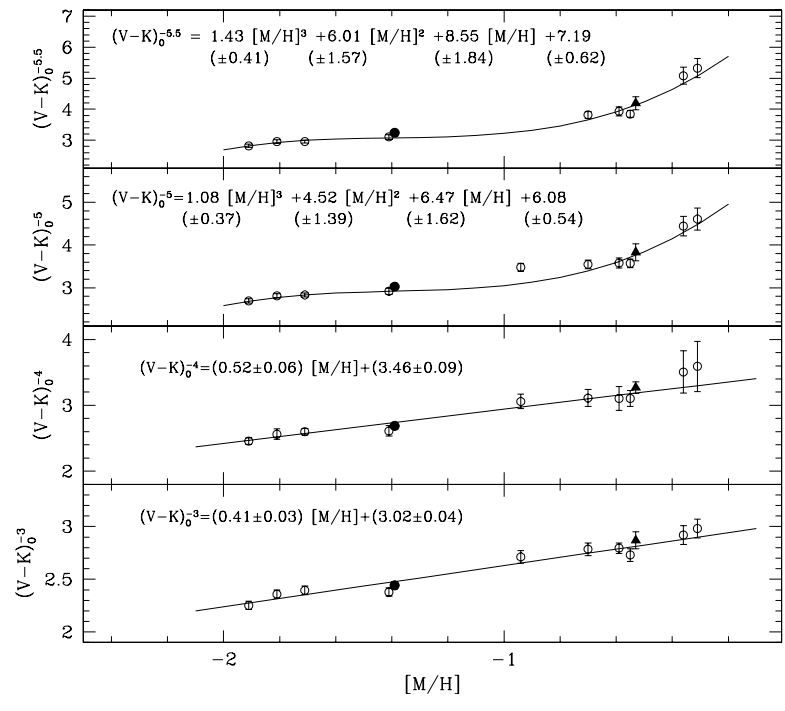

Fig. 8. RGB mean $(V-K)_{0}$ colors at different magnitudes $\left(M_{K}=\right.$ $-3,-4,-5,-5.5)$ as a function of global metallicity for the ten GGCs of F00 (open circles), the RGB-MP (filled circle) and the RGB-a (filled triangle).

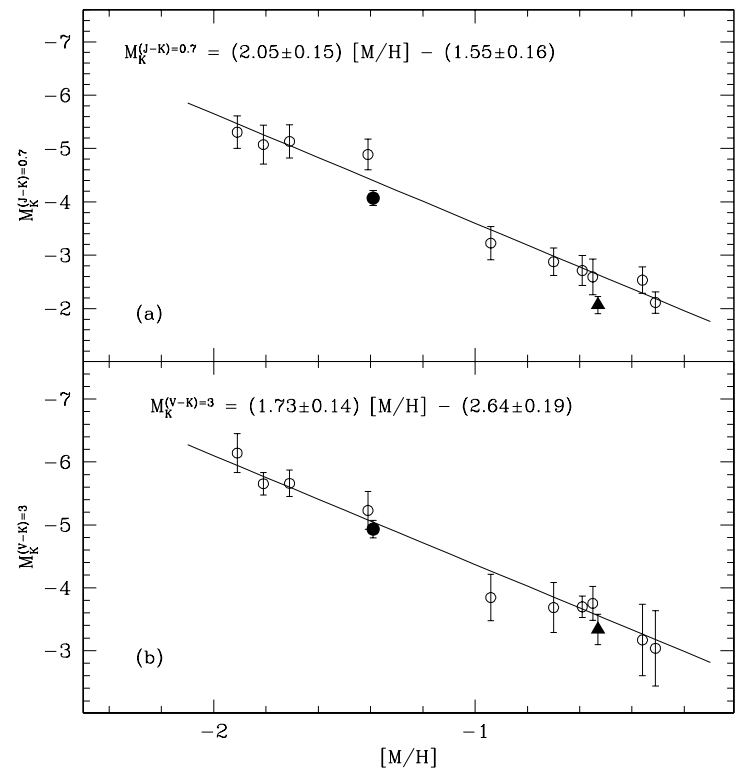

Fig. 9. $M_{K}$ at constant $(J-K)_{0}=0.7$ (panel a)) and $(V-K)_{0}=3$ (panel $\mathbf{b})$ ) as a function of global metallicity for the ten GGCs of F00 (open circles), the RGB-MP (filled circle) and the RGB-a (filled triangle).

instead clearly underestimated. This discrepancy can be due to uncertaintes in the reddening assumptions: in fact errors of a few hundredths of a magnitude produce uncertainties of about $0.2-0.3$ in $\mathrm{K}$, depending on the RGB region intercepted.

To further describe the properties of the RGBs of $\omega$ Cen, we have measured the RGB slope, adopting the technique described by Kuchinski et al. (1995) and using the RGB samples described in Sect. 3 and shown in Fig. 5. Following the prescriptions by Kuchinski et al. (1995), only the brightest portion of the RGB ( $\sim 1$ mag above the HB level) has been considered in the fit. As already emphasized by F00, although linear fits are not the best representation of the RGB shape, the measurement 


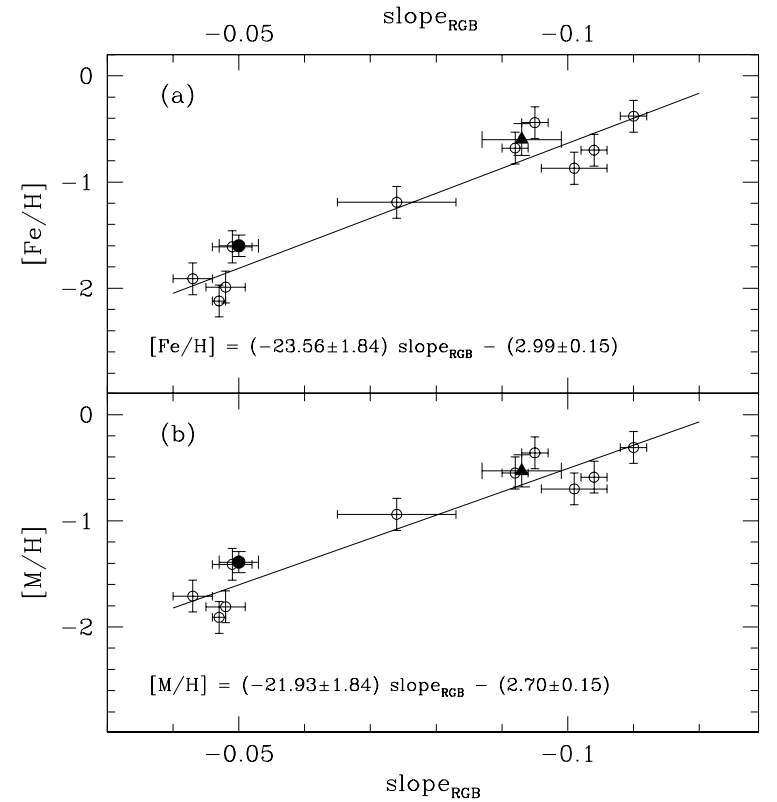

Fig. 10. Metallicity scales: $[\mathrm{Fe} / \mathrm{H}]($ panel a)) and $[\mathrm{M} / \mathrm{H}]($ panel b)) as a function of the derived RGB slope for the ten GGCs of F00 (open circles), the RGB-MP (filled circle) and the RGB-a (filled triangle).

Table 7. RGB magnitudes and slopes for the RGB-MP and RGB-a of $\omega$ Cen.

\begin{tabular}{cccc}
\hline \hline & $M_{K}^{(J-K)_{0}=0.7}$ & $M_{K}^{(V-K)_{0}=3}$ & Slope $_{\mathrm{RGB}}$ \\
\hline RGB-MP & $-4.00 \pm 0.14$ & $-4.93 \pm 0.14$ & $-0.050 \pm 0.003$ \\
RGB-a & $-2.07 \pm 0.16$ & $-3.34 \pm 0.24$ & $-0.093 \pm 0.006$ \\
\hline
\end{tabular}

of the RGB slope is still important since it represents a distance and reddening independent parameter to describe the RGB morphology. The inferred slopes for the RGB-MP and the RGB-a are consistent, within the uncertainties, with those of clusters of similar metal content (see Fig. 10). The adopted absolute $M_{K}$ magnitudes at $(V-K)_{0}=3$ and $(J-K)_{0}=0.7$ and the slopes of the RGB for the two main populations of $\omega$ Cen are listed in Table 7.

\subsection{The theoretical plane}

The transformation of the RGB observed colors and magnitudes onto the theoretical plane $\left(M_{\mathrm{Bol}}, \log T_{\mathrm{e}}\right)$ has been performed using the bolometric corrections and temperature scale for Population II giants computed by Montegriffo et al. (1998). In Fig. 11 the fiducial lines for the two populations of $\omega$ Cen are compared to a sample of GGCs from F00 in the theoretical plane. From this diagram, we can easily derive the RGB effective temperature at a given bolometric magnitude. Figure 12 shows the effective temperature at $M_{\mathrm{Bol}}=-3$ as a function of metallicity for the RGB-MP, the RGB-a and for the sample of clusters of F00. We find a good consistency between the data and the empirical relation of F00.

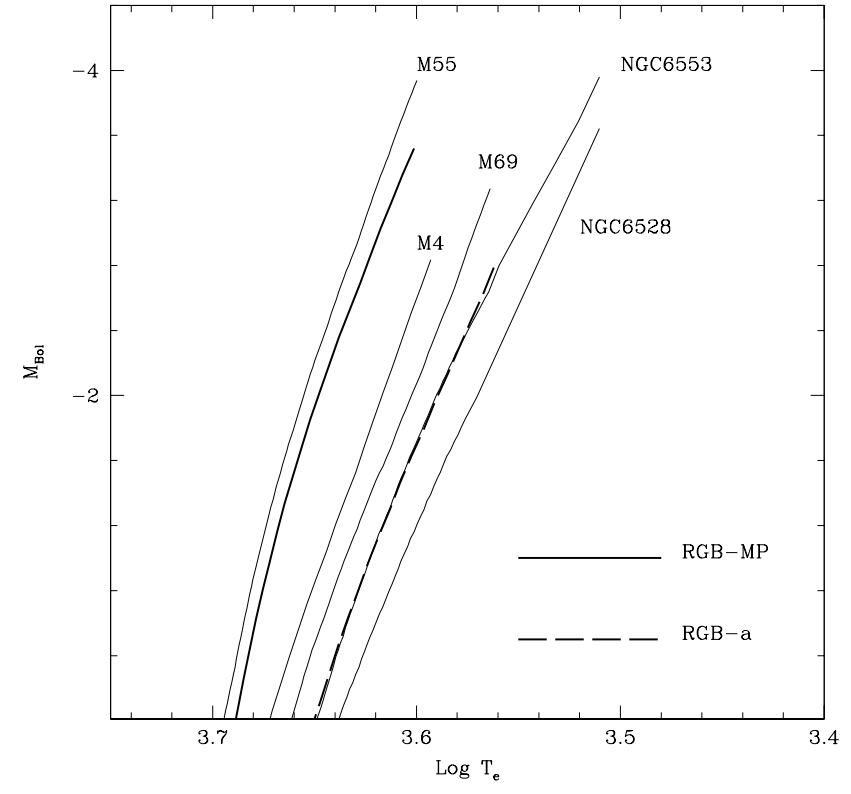

Fig. 11. RGB fiducial ridge lines in the $M_{\mathrm{Bol}}, \log T_{\mathrm{e}}$ theoretical plane for five GGCs of F00 and for the two populations of $\omega$ Cen.

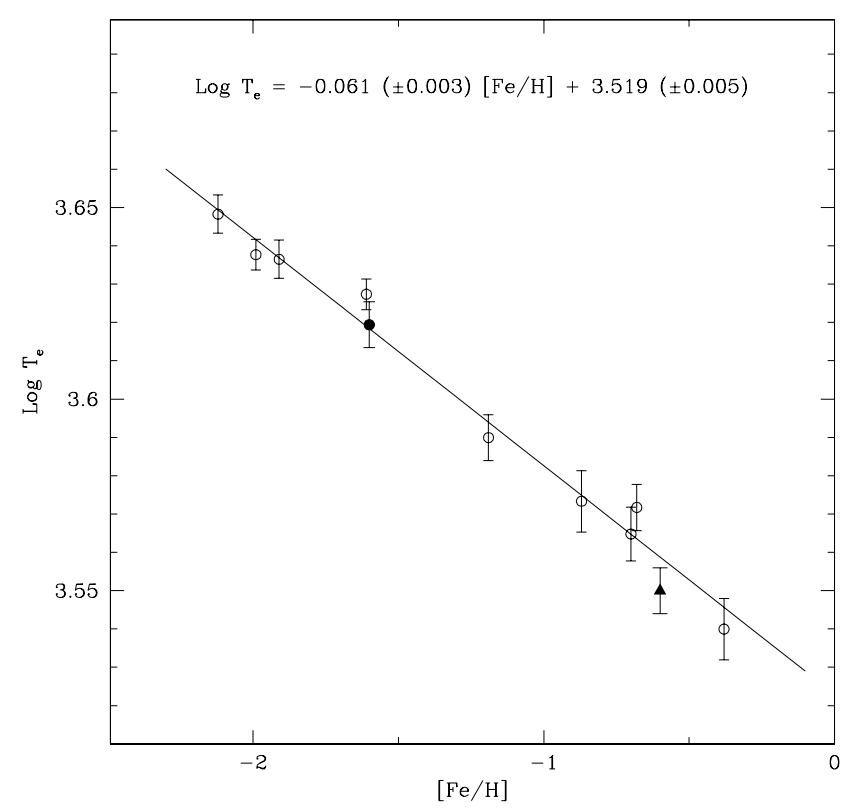

Fig. 12. $\log T_{\mathrm{e}}$ at $M_{\mathrm{Bol}}=-3$ as a function of $[\mathrm{Fe} / \mathrm{H}]$ for the ten GGCs of F00 (open circles), RGB-MP (filled circle) and the RGB-a (filled triangle).

\section{The RGB bump}

To enlarge our sample, both in size and in covered area, we correlated our catalog with that obtained in the external region of the cluster by the 2 MASS survey, which extends to a very wide area of $3^{\circ} \times 2^{\circ}$. Using 1419 common stars, we verified the absence of any significant magnitude offset (see Sect. 2), and then constructed an extended catalog where our magnitudes were maintained in the overlapping regions, while in the outer regions $\left(r>6^{\prime}\right)$ the 2MASS catalog objects were added. We thus obtained $J$ and $K$ magnitudes for a global sample of more than 120000 stars which allowed us to identify with good accuracy 


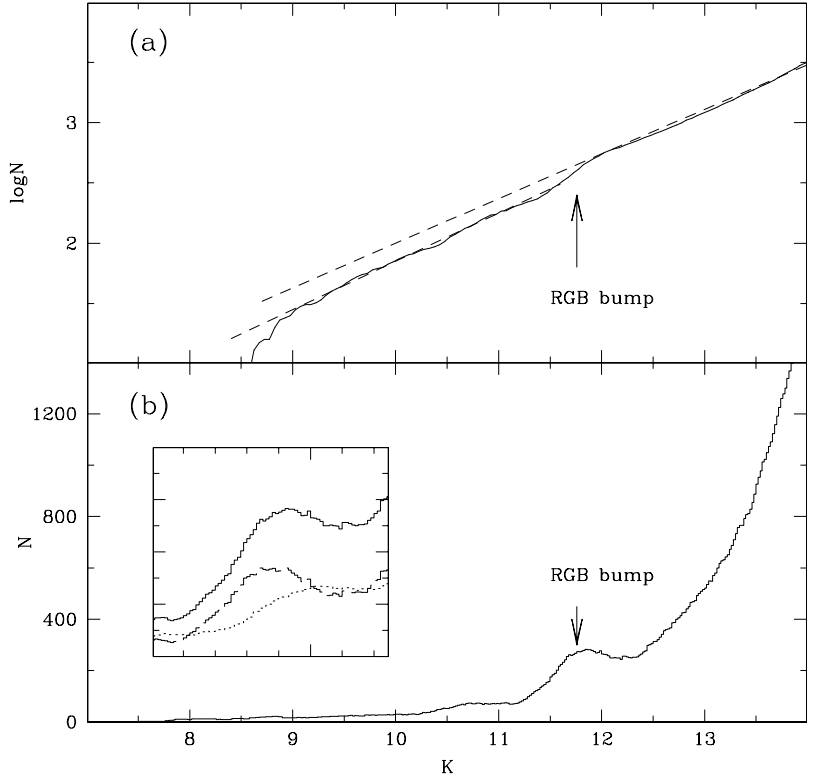

Fig. 13. $K$-band cumulative (panel a)) and differential (panel b)) luminosity functions of the RGB-MP, shown as smoothed histograms. The slope variation and the RGB bump location have been marked. The contribution of the redder part of the RGB (dotted line) and of the bluer part (dashed line) to the bump is also shown in the small panel.

one of the most subtle features along the RGB: the RGB bump (Iben 1968).

The identification of the RGB bump is not an easy task because of the need of large observational samples (Crocker \& Rood 1984; Fusi Pecci et al. 1990). Moreover, since in metal poor clusters the RGB bump occurs at brighter luminosities, in a region that is intrinsically poorly populated, its identification is even more difficult. To correctly locate the bump we used both the cumulative and differential RGB luminosity functions (LF), by detecting the slope variation in the former and identifying the corresponding peak in the latter (Fusi Pecci et al. 1990). We identified the RGB-MP bump with good accuracy both in the $K$-band and in the $V$-band (using the optical catalogue of Pancino et al. 2000) at the observed magnitudes $V_{\text {Bump }}=14.40 \pm 0.05$ and $K_{\text {Bump }}=11.76 \pm 0.05$. The absolute values turn out to be $M_{V}^{\text {Bump }}=0.41 \pm 0.14$ and $M_{K}^{\text {Bump }}=-1.93 \pm 0.14$, with the adopted distance modulus and reddening corrections.

Our detection of the RGB bump is illustrated in Fig. 13, where the LF of the RGB-MP in the $K$ band is shown as a smoothed histogram. In Fig. 14 the absolute $K$ magnitude of the bump is compared with the empirical F00 relation and with the theoretical models by Straniero et al. (1997). The location of the RGB-MP bump is in reasonable agreement with the predictions of both relations. However, the shape of the bump on the differential LF appears widened, as can be expected due to a possible residual contamination of RGB-MInt stars in our RGB-MP sample. To test this hypothesis, we have compared in Fig. 13 the position of the RGB-MP bump with the bump position computed for the blue side of the RGB-MP and the red side of the RGB-MP, separately. As expected, the red side bump appears shifted to fainter magnitudes while the blue side bump

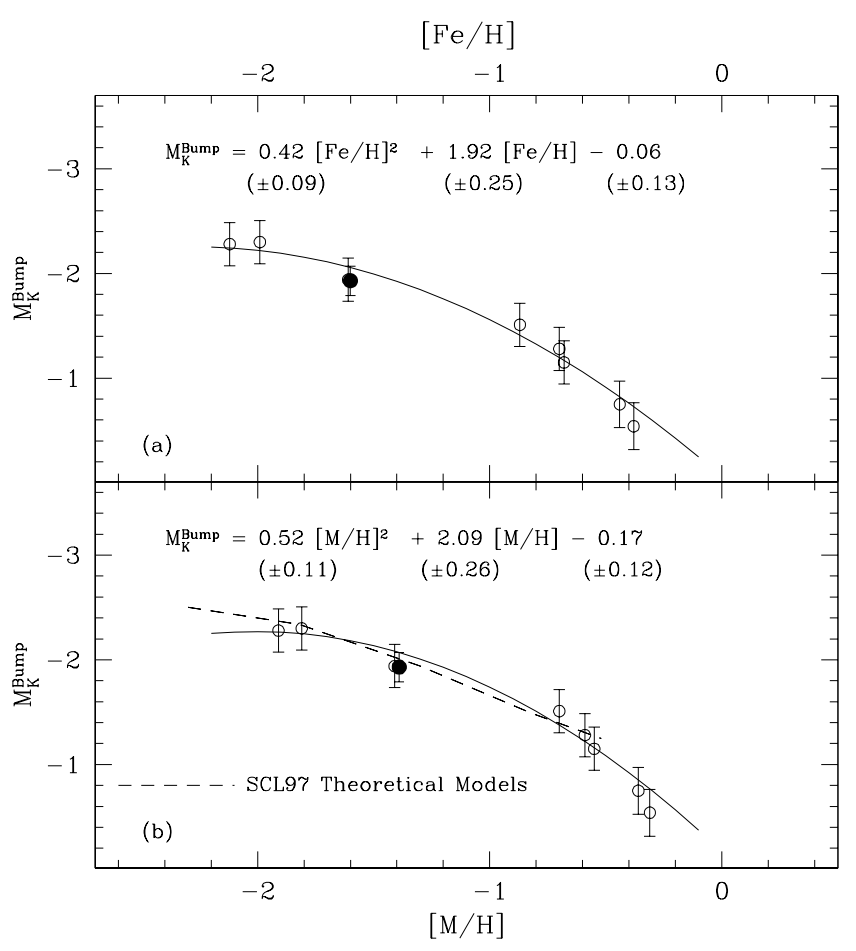

Fig. 14. $M_{K}$ at the RGB bump as a function of the metallicity $[\mathrm{Fe} / \mathrm{H}]$ (panel a)) and on the global scale (panel b)) for 8 GGCs of F00 (open circles) and the RGB-MP (filled circles). The dashed line in panel b) is the theoretical prediction by Straniero et al. (1997) models at $t=16 \mathrm{Gyr}$.

appears less widened and slightly shifted towards brighter magnitudes. The presence of multiple bumps at different magnitudes for different sub-samples of the RGB has been shown also by Rey et al. (2004, see their Fig. 10), in agreement with what is found here.

\section{Conclusion}

We presented an extensive near IR, $J$ and $K$ catalog of stars in the giant globular cluster $\omega$ Cen. More than 73000 stars have been measured allowing an accurate photometric characterization of the RGB. In particular, the colors at different magnitude levels, the magnitude at different colors, the RGB slope and the RGB bump position have been measured, scaled to the absolute plane and compared to similar features measured in clusters with different metallicities by F00. The agreement with the F00 relations is quite good, and the photometric properties of the newly discovered anomalous RGB (RGB-a) consistently reflect the high metal content of this sub-population found by previous spectroscopic work.

Acknowledgements. We warmly thank Paolo Montegriffo for assistance during the catalogs cross-correlation and astrometric calibration procedure. We also thank Katia Cunha, the referee of our paper, for her precious comments and suggestions. The financial support of the Agenzia Spaziale Italiana and the Ministero della Istruzione e della Ricerca Universitaria is kindly acknowledged. This publication makes use of data product from the Two Micron All Sky Survey, which is a joint project of the University of Massachussets and the Infrared Processing Data Analysis Center/California Institute of Technology, 
founded by the National Aeronautics, the Space Administration and the National Science Foundation.

\section{References}

Bellazzini, M., Ferraro, F. R., \& Pancino, E. 2001, ApJ, 556, 635

Buonanno, R., Buscema, G., Corsi, C. E., Ferraro, I., \& Iannicola, G. 1983, A\&A, 126, 278

Buonanno, R., \& Iannicola, G. 1988, PASP, 108, 294

Caputo, F., Degl'Innocenti, S., \& Marconi, M. 2002, in A unique window into Astrophysics, ASP Conf. Ser.

Crocker, D. A., \& Rood, R. T. 1984, in The Observational Tests of the Stellar Evolution Theory, ed. A. Maeder, \& A. Renzini (Dordrecht: Reidel), 159

D'Cruz, N., O'Connell, R., Rood, R., et al. 1999, upse. conf., 13

Dickens, R. J., \& Bell, R. A. 1976, ApJ, 207, 506

Ferraro, F. R., Fusi Pecci, F., Guarnieri, A., et al. 1994, MNRAS, 266, 829

Ferraro, F. R., Fusi Pecci, F., Testa, V., et al. 1995, MNRAS, 272, 391

Ferraro, F. R., Messineo, M., Fusi Pecci, F., et al. 1999 AJ, 118, 1738

Ferraro, F. R., Montegriffo, P., Origlia, L., \& Fusi Pecci, F. 2000, AJ, 119,1282

Ferraro, F. R., Pancino, E., \& Bellazzini, M. 2001, in A Unique Window into Astrophysics, 2002, ASP Conf. Ser.

Ferraro, F. R., Bellazzini, M., \& Pancino, E. 2002, ApJ, 573, 95

Fusi Pecci, F., Ferraro, F. R., Crocker, D., Rood, R. T., \& Buonanno, R. 1990, A\&A, 238, 95

Glass, I. S., \& Feast, M. W. 1973, MNRAS, 163, 245

Glass, I. S., \& Feast, M. W. 1977, MNRAS, 181, 509

Kuchinski, L. E., Frogel, J. A., Terndrup, D. M., \& Persson, S. E. 1995, ApJ, 109, 1131

Iben, I. Jr. 1968, Nature, 220, 143

Lee, Y. W., Joo, J. M., Sohn, Y. J., et al. 1999, Nature, 402, 55
Lub, J. 2002, in A Unique Window into Astrophysics, ASP Conf. Ser. Montegriffo, P., Ferraro, F. R., Origlia, L., \& Fusi Pecci, F. 1998, MNRAS, 297, 872

Norris, J. E., \& Da Costa, G. S. 1995, ApJ, 447, 680

Norris, J. E., Freeman, K. C., \& Mighell, K. J. 1996, ApJ, 462, 241

Origlia, L., Ferraro, F. R., Rood, R., \& Fusi Pecci, F. 2002, ApJ, 571, 458

Origlia, L., Ferraro, F. R., Bellazzini, M., \& Pancino, E. 2003, ApJ, 591,9160

Pancino, E., Ferraro, F. R., Bellazzini, M., Piotto, G., \& Zoccali, M. 2000, ApJ, 534, L83

Pancino, E., Pasquini, L., Hill, V., Ferraro, F. R., \& Bellazzini, M. 2002, ApJ, 568, L101

Pancino, E., Seleznev, A., Ferraro, F. R., Bellazzini, M., \& Piotto, G. 2003, MNRAS, 345, 683

Persson, S. E., Frogel, J. A., Cohen, J. G., Aaronson, M., \& Matthews, K. 1980, AJ, 235, 452

Persson, S. E., Murphy, D. C., Krezminski, W., Roth, M., \& Rieke, M. J. 1998, AJ, 116, 2475

Pulone, L., de Marchi, G., Paresce, F., \& Allard, F. 1998, ApJ, 492, L41

Rey, S.-C., Lee, Y.-W., Ree, C. H., et al. 2004, AJ, 127, 958

Salaris, M., Chieffi, A., \& Straniero, O. 1993, Mem. Soc. Astron. Italiana, 63, 315

Savage, B. D., \& Mathis, J. S. 1979, ARA\&A, 17, 73S

Smith, V. V., Suntzeff, N. B., Cunha, K., et al. 2000, AJ, 119, 1239

Straniero, O., \& Chieffi, A. 1991, ApJS, 76, 911

Straniero, O., Chieffi, A., \& Limongi, M. 1997, ApJ, 490, 425

Suntzeff, N. B., \& Kraft, R. P. 1996, AJ, 111, 1913

Thompson, I. B., Kaluzny, J., Pynch, W., et al. 2001, AJ, 121, 3089

van Leeuwen, F., Le Poole, R. S., Reijns, R. A., Freeman, K. C., \& de Zeeuw, P. T. 2000, A\&A, 360, 472 